\title{
A Comparison of the BEM and RANS Calculations for the hydrodynamic performance of the PODS
}

\author{
Reza Shamsia ${ }^{a}$, Hassan Ghassemi and Mehdi Iranmanesh \\ Department of Maritime Engineering, Amirkabir University of Technology, Tehran, Iran
}

Received 24 August 2015, Accepted 19 September 2016

\begin{abstract}
In the present study, the hydrodynamic performance of the Podded Drive System (PODS) using Reynolds-Averaged Navier Stokes (RANS) method and Boundary Element Method (BEM) is reported. The BEM is based on potential inviscid flow problem while RANS are for the viscous flow problem. Hydrodynamic characteristics of the single propeller are determined by both methods and the results are compared and discussed. Then, it is extended to the PODS at the zero yaw angle. Validation is made through the comparison of both numerical results against experimental test data for the PODS propulsive characteristics coefficients. The results show that there is a good agreement between the experimental data and numerical results, particularly in the viscous calculations.
\end{abstract}

Key words: RANS method / BEM method / Podded drive system / Hydrodynamic forces

\section{Introduction}

PODS is a marine electrical drive system including the pod, the strut and the propeller and electrical motor inside the pod (Fig. 1). Recently, this system is widely used in the marine industry and becoming more popular, not only for commercial ships but also for offshore drilling units and naval vessels. In this system, an electrical motor is located in a steerable pod housing, which drives a fixed-pitch propeller. The total unit is hung below the stern of the ship by the strut and can be rotated through 360 degrees around its vertical axis. Therefore, the thrust is generated in any direction that gives the better maneuverability. This device combines propulsion and maneuvering functions mutually [1].

The prediction of the hydrodynamic forces of the PODS can be important and crucial from structural and operational aspects. For instance, bearing failure is one of the main operational problems of the PODS and occurs when there are significant fluctuations in forces and moments on the PODS. It is a common practice to perform a model test to evaluate the hydrodynamic performance of a marine propeller or propulsion system. However, the model test is usually expensive and time-consuming. Besides, experimental measurements of some parameters are impossible or difficult to achieve. Therefore, finding an efficient and accurate numerical method is strongly desirable. In order to study the performance of the PODS,

${ }^{a}$ Corresponding author: gasemi@aut.ac.ir

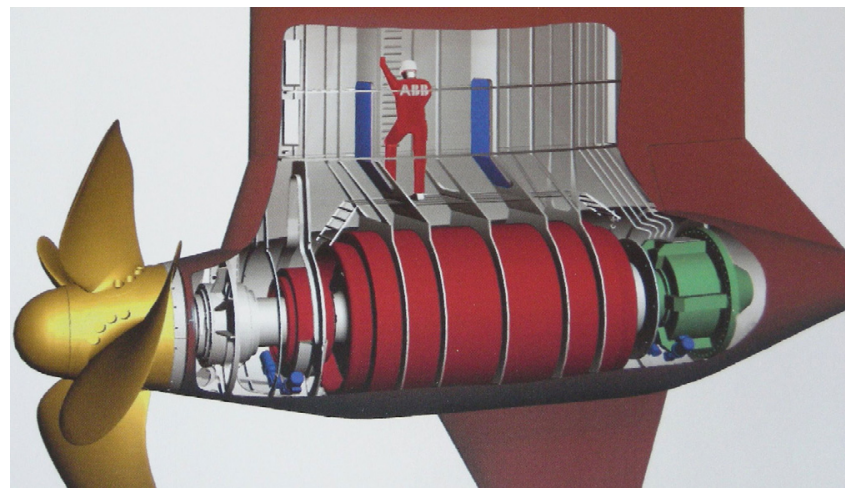

Fig. 1. PODS (included Propeller, pod, strut, electrical motor).

different numerical methods have been used from potential method or hybrid potential/viscous method to pure viscous method [2].

The numerical methods based on potential flow theory have been widely and successfully used to predict the performance of conventional propellers. BEM is an appropriate numerical scheme and a powerful tool for hydrodynamic analysis of the marine propellers, and many researchers have thus far obtained acceptable results using this method. Ghassemi and Ghadimi [3], and Islam et al. [4] predicted propeller and PODS performance using potential flow method. Also, Liu et al. [5] applied unsteady panel method code for predicting unsteady forces, 


\section{Nomenclature}

\begin{tabular}{|ll|}
\hline$D$ & Propeller diameter \\
$f_{x}$ & Axial force \\
$f_{y}$ & Side force \\
$J$ & Propeller advance ratio \\
$K_{Q}$ & Propeller torque coefficient \\
$K_{T}$ & Propeller thrust coefficient \\
$K_{f x}$ & Axial force coefficient \\
$K_{f y}$ & Side force coefficient \\
$n$ & Propeller angular velocity (rps) \\
$\vec{n}$ & Unit normal vector \\
$P$ & Static pressure \\
$Q$ & Propeller torque \\
$T$ & Propeller thrust \\
$S_{B}$ & Body surface \\
$S_{W}$ & Wake surface \\
$S_{\infty}$ & Outer control surface \\
$\vec{V}_{A}$ & Advance velocity \\
$\vec{V}_{I}$ & Inflow velocity \\
$\vec{v}$ & Tangential velocity vector \\
$\delta_{i j}$ & Kronecker delta \\
$\eta$ & Propeller efficiency \\
$\mu$ & Molecular viscosity \\
$\rho$ & Fluid density \\
$\overline{\bar{\tau}}$ & Stress tensor \\
$\omega$ & Angular velocity \\
$\Phi$ & Total velocity potential \\
$\varphi$ & Perturbation velocity potential \\
$\Gamma$ & Circulation \\
\hline
\end{tabular}

torques and bending moments for a podded propulsor unit model at various yaw angles. Inviscid methods offer many advantages over viscous methods such as lower computational time and moderate grid generation. Alternatively, inviscid flow solvers have a limitation in terms of the correct prediction of the hydrodynamic performance of the PODS. In PODS unit, viscous effects cannot be ignored and have to be modeled in order to deliver proper values for propulsive characteristics. Furthermore, the drag forces of the propeller, the pod, and the strut increase highly at large yaw angles due to flow separation.

Viscous solvers are capable of taking the viscous and turbulence effects for PODS into account. The application of viscous models in the PODS has been carried out by Guo et al. [6]. Furthermore, Amini et al. [7], Arikan et al. [8] and Shamsi et al. [9, 10] have studied at the steady and unsteady RANS solvers in order to simulate the flow around the PODS.

The objective of the present study is an evaluation of the capabilities and limitations existing in the numerical methods (RANS and BEM) for predicting the propulsive performance of the PODS. Therefore, a finite volume based RANS solver (commercial software ANSYS Fluent 14.5) and an in-house BEM code are applied to predict the hydrodynamic performance of single propeller and PODS. By these simulations, hydrodynamic charac-

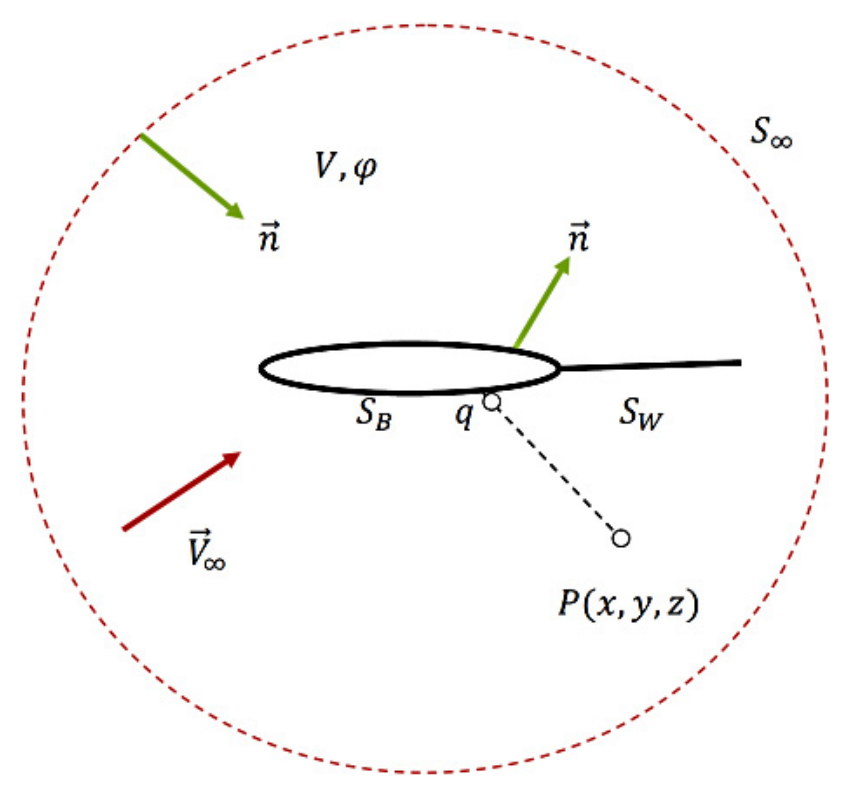

Fig. 2. Demonstration of the Green's theorem on the body.

teristics of a single propeller and PODS are presented, including pressure distribution and open water performance curves. The computational results are discussed and compared with the experimental data.

\section{Mathematical formulae of the BEM}

In BEM, using the Green's theorem, the velocity potential at every point can be expressed as an integral equation in all parts of the boundary and subsequently the potential field is determined at each point. Using this method, the surfaces of the propeller, pod, strut and the trailing vortex surface (helical for a propeller) are divided into hyperboloid-shaped elements. By considering a dipole and a source at each element and solving the resulting system of equations, the potential and pressure fields are determined. Furthermore, the thrust and torque of the propeller are calculated. Procedures and the main tasks involved in this method include grid generation (element generation), calculation of the influence coefficient of the source and doublet singularities at every element along with simultaneous solutions of the equations involving the singularity strength, local velocities, total pressure, forces, and torques.

Consider the closed region $V$ with boundary $S$ and the unit vector of $\vec{n}$ normal to $S$ as depicted in Figure 2 . Boundary $S$ of the flow region includes the surface of the body $S_{B}$, the surface of the wake $S_{\mathrm{W}}$, and the outer control surface $S_{\infty}$ that surrounds the surfaces of the body and the trailing vortex surface. Based on the assumption that the fluid in region $V$ is incompressible, inviscid, and irrotational, region $V$ is subjected to the inflow uniform velocity $\vec{V}_{I}$ at the upstream of the flow. With these assumptions, the flow field around the body is characterized by the total velocity potential $\Phi$ and perturbation velocity 


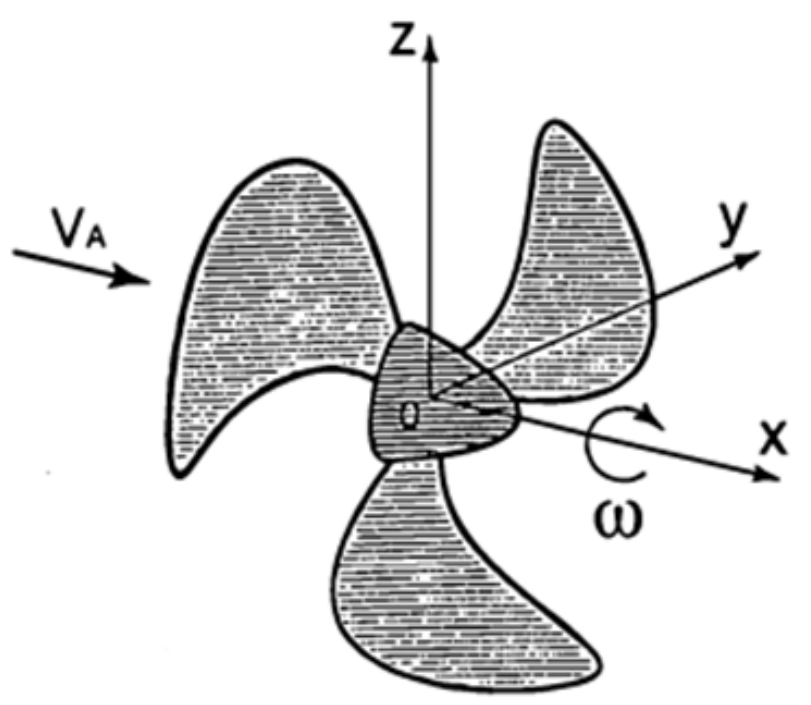

Fig. 3. Coordinate system of the propeller.

potential $\varphi$ which satisfies Laplace's equations:

$$
\left\{\begin{array}{l}
\nabla^{2} \varphi=0 \\
\nabla^{2} \Phi=0
\end{array}\right.
$$

In Equation (1), the total velocity potential is defined as follows:

$$
\begin{aligned}
\Phi & =\varphi+\varphi_{I} \\
\varphi_{I} & =V_{I} X
\end{aligned}
$$

where $X$ is the position on the propeller surface. The boundary value problem (BVP) is constructed by identifying three boundary conditions on the boundary $S$, as described below [11]:

(a) Tangential condition: The kinetic boundary condition requires that the total flow velocity perpendicular to the body surface $S_{\mathrm{B}}$ should be equal to zero:

$$
\frac{\partial \varphi}{\partial n}=-\vec{V}_{I} \cdot \vec{n}
$$

where $\vec{n}$ is the unit normal vector, pointing out of the boundary. The inflow velocity to the propeller can be expressed as follows:

$$
\vec{V}_{I}(X, Y, Z)=\vec{V}_{A}(X, Y, Z)+\vec{\omega} \times \vec{r}(X, Y, Z)
$$

where $\vec{V}_{A}(X, Y, Z)$ is advance velocity from the ship's propeller, as shown in Figure 3.

(b) Conditions on the trailing vortex surface: No flow velocity jump occurs on the trailing vortex surface, but velocity potential jump does occur on this surface and is equal to the circulation $\Gamma$ around the blade. Mathematical equations of these two conditions (i.e. the flow velocity and velocity potential) are expressed as follows:

$$
\begin{aligned}
(\Delta \varphi)_{o n S_{W}} & =\varphi^{B}-\varphi^{F}=\Gamma \\
\left(\Delta \frac{\partial \varphi}{\partial n}\right)_{o n S_{W}} & =\left(\frac{\partial \varphi}{\partial n}\right)^{B}-\left(\frac{\partial \varphi}{\partial n}\right)^{F}=0
\end{aligned}
$$

where $B$ and $F$ indicate the back side and face side of the propeller.

(c) Kutta condition at the trailing edge (TE): This condition is one of the most important conditions used for the lifting surfaces. The Kutta condition theory indicates that the velocity would remain limited along the sharp trailing edge. As another equivalent form, it can be understood that the pressures on both back and face sides of the blade are identical. This Kutta condition of equal pressure is used for determining the unknowns $\Delta \varphi$ of the dipole strength for a trailing vortex surface. It is defined as:

$$
|\nabla \varphi|_{\text {T.E. }}<0
$$

Using Green's theorem, the perturbation velocity potential at each point of the field can be expressed as an integral equation corresponding to the distribution of the source and dipole. Therefore, for a field point $p$ in region $\mathrm{V}$ [3], we write:

$$
\begin{aligned}
& 2 \pi \varphi(P)=\iint_{S_{B}} \varphi(q) \frac{\partial}{\partial n_{q}} \frac{1}{R(p, q)} \mathrm{d} s \\
& -\iint_{S_{B}} \frac{\partial \varphi(q)}{\partial n_{q}} \frac{1}{R(p, q)} \mathrm{d} s+\iint_{S_{W}} \Delta \varphi(q) \frac{\partial}{\partial n_{q}} \frac{1}{R(p, q)} \mathrm{d} s
\end{aligned}
$$

where $R(p, q)$ is the distance between the singular point $P(x, y, z)$ and the point $q(u, v, w)$. The intrinsic coordinate $(u, v, w)$ is defined for the $q$ where the integral is calculated. The right hand of the Equation (9) has three terms. The first two terms are the dipole and source on the body while the third term is the dipole on the trailing vortex wake surface (defined only for lifting bodies). This equation can be considered as an illustration of the velocity potential corresponding to the distribution of a dipole strength of $\varphi$ on the surface of the body $S_{B}$, the distribution of the source strength $\partial \varphi / \partial n$ on $S_{B}$, and the distribution of dipole strength of $\Delta \varphi$ on the trailing vortex surface $S_{W}$. At this point, the only remaining unknown is the dipole strength $\varphi$ which is obtained by simultaneous solution of Equations (7) and (8). The term $\partial \varphi / \partial n$ in Equation (9) is obtained from the boundary condition (a) in Equation (2).

For obtaining a numerical solution for the integral equation, the surfaces of the propeller blades, hub, pod, strut, and trailing vortex are divided into small elements. In the usual applications of the boundary element methods for the analysis of the propeller, quadrilateral plane elements are used for surface approximation. Generally, selection of element type and the generation of smooth elements are important parts of this method as is in all other numerical methods in which the choices may affect the results directly. This implies that the solver may operate properly while the generation of an improper grid may generate numerical errors in the computed results.

In the pusher PODS, the propeller operates at the downstream of the pod and strut where the inflow is nonuniform due to the wake generated by them. At this condition, all the blades should be considered in the computation. For the propeller with $Z$ number of blades and $M$ 


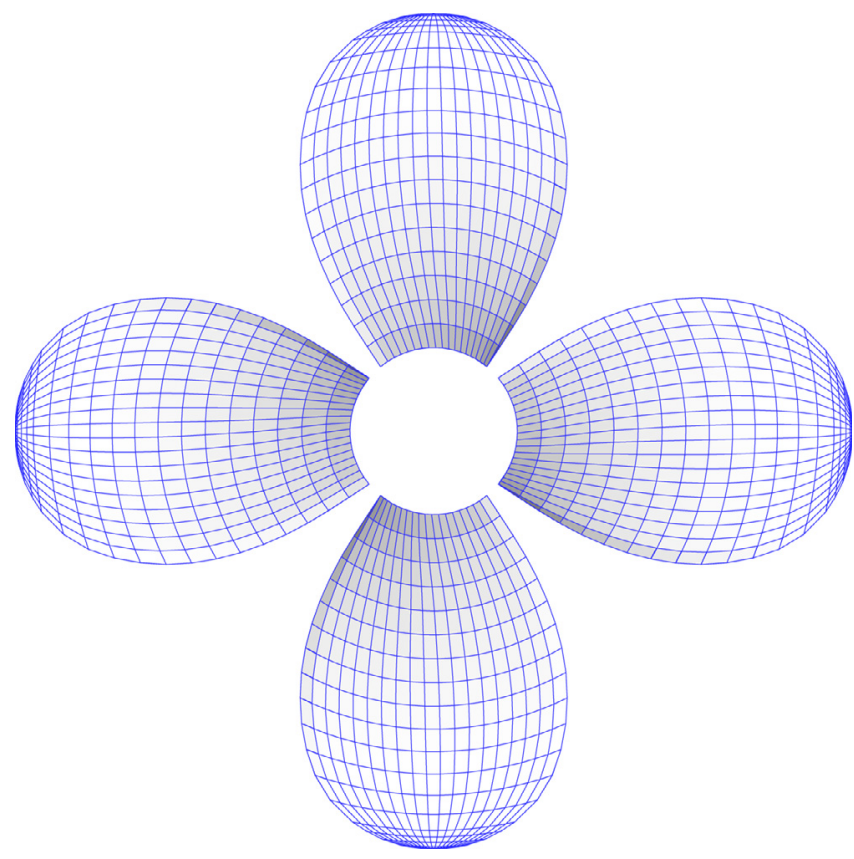

Fig. 4. Propeller panel arrangement.

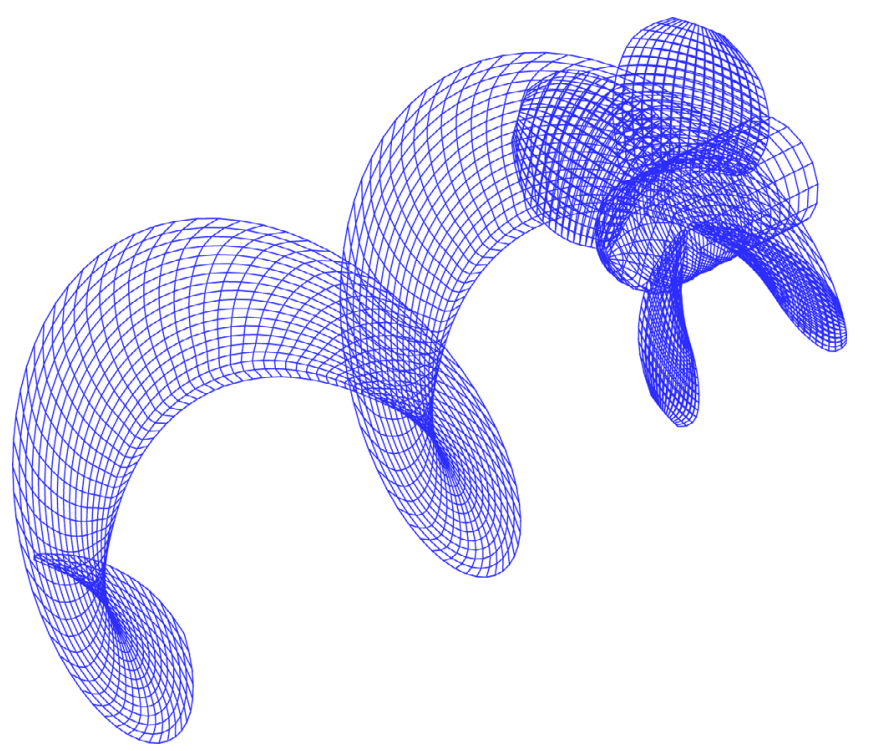

Fig. 5. Propeller panel arrangement with wake grid.

and $N$ divisions in the radial as well as chordwise directions, respectively, the total number of blades elements is equal to $2 \times N \times M \times Z$. In this study, the propeller blades are discretized with 19 chordwise and 19 spanwise panels, as shown in Figure 4. As such, 2888 elements are generated on a four-bladed propeller and 400 elements are generated on the hub; also a total of 644 elements are generated for the pod and strut.

Figure 5 shows the panel arrangement of the propeller blade with an open-water hub and the prescribed wake grid. The modeling of the trailing vortex wake is important in the BEM to employ the Kutta condition. The propeller blade is helical and when it is rotating, a classical wake model can be generated. Because of the propeller is going to ahead and this wake is shed from the trailing edge. A prescribed wake grid in the form of the classical helicoidal model with the pitch of the helicoidal lines equal to the propeller pitch is used. This wake is generated for propeller cycle (one turning of the propeller) [12].

\section{Governring equations of the RANS method}

In this study, it is assumed that the fluid is incompressible, the tip Mach number is less than 0.15. The governing equations are the mass and momentum conservations. Using the Reynolds averaging approach, the Navier-Stokes equations can be stated as:

$$
\begin{aligned}
\frac{\partial u_{i}}{\partial x_{i}} & =0 \\
\frac{\partial}{\partial t}\left(\rho u_{i}\right)+\frac{\partial}{\partial x_{j}}\left(\rho u_{i} u_{j}\right) & =-\frac{\partial p}{\partial x_{i}}+\frac{\partial}{\partial x_{j}}\left(\mu \frac{\partial u_{i}}{\partial x_{j}}-\rho \overline{u_{i}^{\prime} u_{j}^{\prime}}\right)
\end{aligned}
$$

where $-\rho \overline{u_{i}^{\prime} u_{j}^{\prime}}$ tensor represents the Reynolds stresses.

It is rather difficult to model the components of the Reynolds stress tensor because it requires detailed and unavailable data about turbulent structures in the flow. When a turbulence model is to be chosen, it is worth considering whether a complicated or a simple model should be used. The $k-\varepsilon$ and $k-\omega$ models are the most widely used turbulence models for external aerodynamics and hydrodynamics analyses. Here, the two-equation standard $k$ - $\omega$ model developed by Wilcox [13] is employed, which incorporates the modifications for low-Reynolds-number effects, compressibility, and shear flow spreading. This model is an empirical model in which one equation involves the turbulence kinetic energy $(k)$ representing the velocity scale, whereas the other takes the turbulent dissipation rate $(\omega)$ into account representing the length scale. The two-equation standard $k$ - $\omega$ turbulence model accounting for the effect of the turbulence can be presented in the following form:

$$
\begin{aligned}
\frac{\partial}{\partial t}(\rho k)+\frac{\partial}{\partial x_{i}}\left(\rho k u_{i}\right) & =\tau_{i j} \frac{\partial u_{i}}{\partial x_{j}} \rho \beta^{*} k \omega \\
& +\frac{\partial}{\partial x_{i}}\left[\left(\mu+\frac{\mu_{t}}{\sigma_{k}}\right)\right] \frac{\partial k}{\partial x_{j}} \\
\frac{\partial}{\partial t}(\rho \omega)+\frac{\partial}{\partial x_{i}}\left(\rho \omega u_{i}\right) & =\alpha \frac{\omega}{k} \tau_{i j} \frac{\partial u_{i}}{\partial x_{j}}-\rho \beta \omega^{2} \\
& +\frac{\partial}{\partial x_{i}}\left[\left(\mu+\frac{\mu_{t}}{\sigma_{\omega}}\right)\right] \frac{\partial k}{\partial x_{j}}
\end{aligned}
$$

where $\sigma_{k}$ and $\sigma_{\omega}$ are the turbulent Prandtl numbers for $k$ and $\omega$, respectively. The turbulent viscosity, $\mu_{t}$, is computed by combining $k$ and $\omega$ as follows:

$$
\mu_{t}=\alpha^{*} \rho \frac{k}{\omega}
$$

The coefficient $\alpha^{*}$ damps the turbulent viscosity causing a low-Reynolds-number correction. 


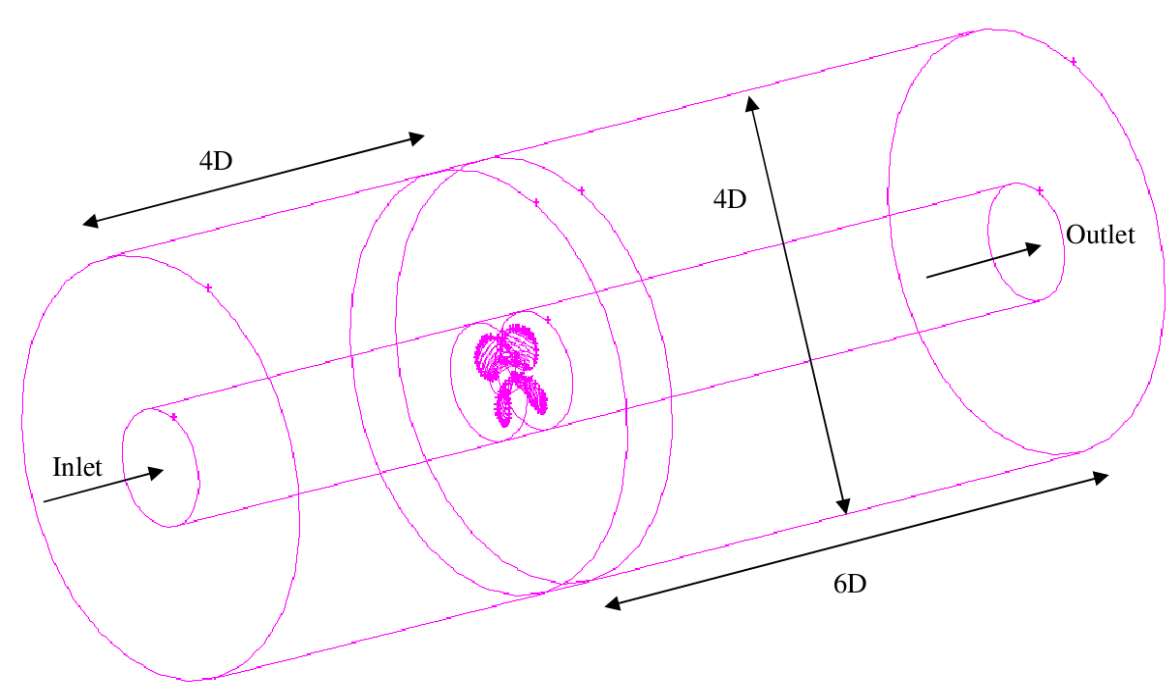

Fig. 6. Model of solution field for single propeller.

\section{Hydrodynamic performance of the propeller and pod}

When a propeller with diameter $(D)$ is operating with angular velocity $\omega(=2 \pi n)$ and advance velocity $\left(V_{A}\right)$, it generates thrust and torque. The hydrodynamic performance characteristics of the propeller can be defined as the non-dimensional coefficients such as the advance coefficient $(J)$, thrust coefficient $\left(K_{T}\right)$, torque coefficient $\left(K_{Q}\right)$ and efficiency $(\eta)$ which can be computed respectively as follows [1]:

$$
J=\frac{V_{A}}{n D}, \quad K_{T}=\frac{T}{\rho n^{2} D^{4}}, \quad K_{Q}=\frac{Q}{\rho n^{2} D^{5}}, \eta=\frac{J}{2 \pi} \frac{K_{T}}{K_{Q}}
$$

For podded propulsors, the coefficients of axial $\left(K_{F x}\right)$ and side force $\left(K_{F y}\right)$ are defined as:

$$
K_{F_{x}}=\frac{F_{x}}{\rho n^{2} D^{4}}, \quad K_{F_{y}}=\frac{F_{y}}{\rho n^{2} D^{4}}
$$

where $F_{x}$ and $F_{y}$ are total forces on the whole unit in $x$ and $y$-directions, respectively. These coefficients are important factors in the design of podded propulsors affecting propulsion and maneuvering specifications because side force is not good for straight course keeping. General speaking, podded propulsor (or better name is the Azimuthing Podded drive $=$ AZIPOD) is not needed to have a rudder, it may be rotated around the vertical axis and can generate thrust in any direction [14].

In RANS method, the fluid domain around the propeller is modeled by means of rotating and fixed cylindrical frames. The rotating frame simulates the propeller rotation and uses the Coriolis acceleration terms in the governing equations for the fluid. In the case of the single propeller, the fixed frame is a circular cylinder with the diameter of $4 \mathrm{D}$, where $D$ is the propeller diameter. The distance between the rotating frame and inlet is nearly
$4 \mathrm{D}$ while it is nearly $6 \mathrm{D}$ between the outlet and dynamic frame. The same method is also employed for the pusher PODS except that the dimensions of frames are selected related to the pod length. The positions of the inlet and outlet are $2.5 L_{\text {Pod }}$ and $5 L_{\text {Pod }}$ respectively; where $L_{\text {Pod }}$ is the pod length. The diameter of the fixed frame is selected as $6 L_{\text {Pod }}$. Figures 6 and 7 demonstrate the computational domain for the single propeller and PODS respectively. Constant inlet velocity profile is assumed as the inlet boundary condition of the fixed frame. The outlet boundary condition can also be set as the constant pressure in this frame. The propeller blades, the pod, the strut, and the fixed cylinder are assumed as no-slip boundary conditions. The RANS simulations are performed in the steady state, not in time-accurate.

In the case of the single propeller, the solution field is divided into six blocks. Thereafter, the rotating block is meshed with unstructured tetrahedral cells, whereas the other blocks are meshed with structured hexahedral cells. In order to obtain an evaluation of the mesh convergence, the grid dependency study is performed. Particularly, this study examines different element sizes. Thus, the best compromise between element size and accuracy has been obtained from the results of this work. Seven models in the same domain with different element sizes and cell numbers are generated. These models are simulated at $J=0.6$ and the results are compared to the available experimental results. The variation of error values for the propeller thrust and torque coefficients are shown in Figure 8. According to this figure, the error is reduced by an increase in the number of the elements. As a result, the surface of the propeller and hub are meshed with the size of $0.005 \mathrm{D}$. Also, four prismatic cells with the size of $0.001 \mathrm{D}$ are considered as a boundary layer on the propeller surface. The number of grids for the single propeller is about 2.2 million. The PODS modeled with ten blocks and unstructured tetrahedral cells is applied in the rotating block and static blocks. In the case of the 


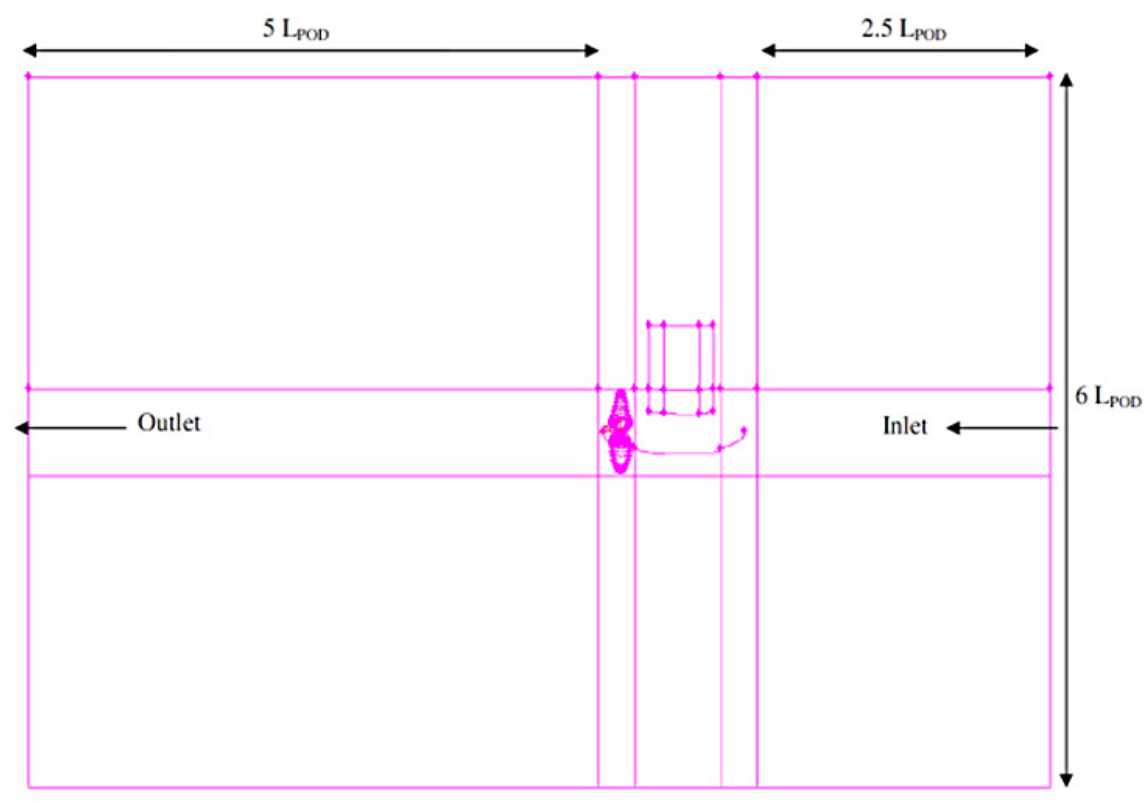

Fig. 7. Model of solution field for the PODS.

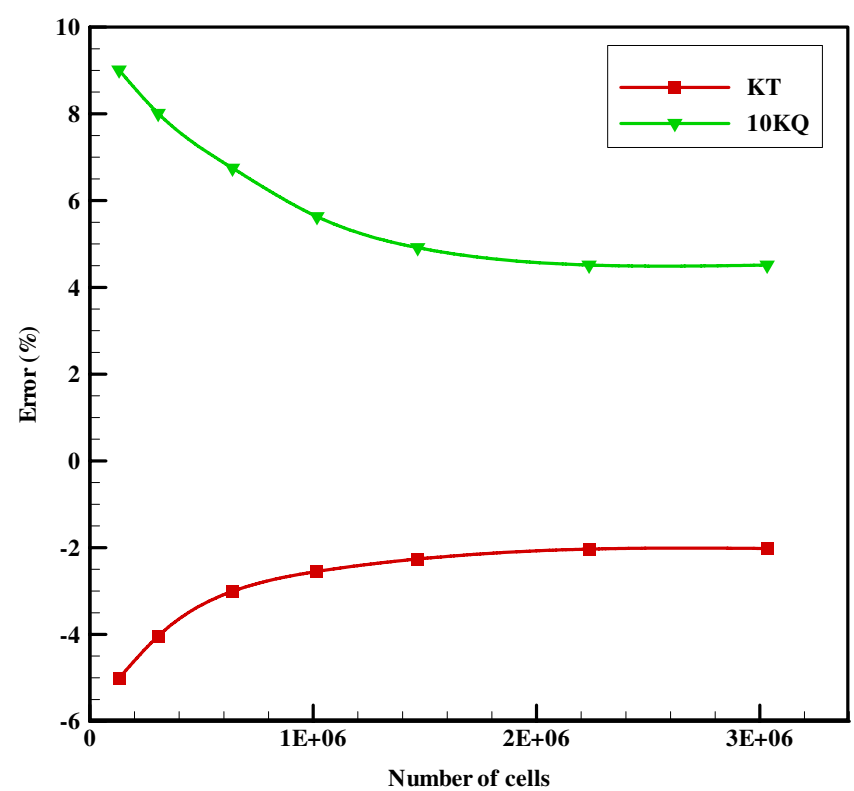

Fig. 8. Variation of error value of thrust and torque coefficients with increasing the number of cells at $J=0.6$.

PODS, the domain is discretized into about 3.1 million cells.

The governing equations of this problem are solved by the finite volume method based on the RANS equations. The ANSYS Fluent 14.5 software is used to solve the RANS equations. The discretized equations are solved using Green-Gauss cell-based gradient option. The SIMPLE algorithm is used for solving the pressure-velocity coupling equations. The second-order upwind discretization scheme is utilized for the momentum, turbulent kinetic energy, and turbulent dissipation rate in this problem.

Monitoring of the convergence of the solution is performed. There are six differential equations to be solved in this three-dimensional incompressible turbulent flow problem. For this purpose, the six residuals i.e. continuity, $x$-velocity , $y$-velocity , $z$-velocity, turbulence kinetic energy $(k)$, and turbulent dissipation rate $(\omega)$ should be monitored for convergence. The default convergence criteria are set as 1e- 6 for all these residuals. The residuals are calculated in each iteration. These residuals represented an average error in the solution.

\section{Results and discussions}

The numerical methods mentioned above are employed for the simulation of the single propeller and the PODS. For the numerical analysis, two different simulations have been carried out. As the first part of the simulation, a single propeller has been analyzed in open water condition and the results are validated with the experimental data (Sect. 4.1). On the other hand, in the second part of the simulation (Sect. 4.2), the numerical analysis has been extended to the total unit of PODS. In both cases, the models are simulated using BEM and RANS method.

At first, a single propeller model with hub taper angles is selected. The model is a four-bladed propeller without skew or rake with the uniform pitch-to-diameter ratio of 1.0. Afterward, a PODS model is utilized for simulation. The main dimensions of the propeller model and PODS are presented in Tables 1 and 2 respectively. The PODS views are shown in Figure 9. It is a generic pod based on the average dimension of commercially available pods. The Cartesian coordinate system is used where 
Table 1. Main dimensions of propeller model.

\begin{tabular}{lc}
\hline Parameters & Value \\
\hline Profile Type & NACA 66 (DTMB Modified) \\
No. of blades $(Z)$ & 4 \\
Diameter $(D)(\mathrm{mm})$ & 270 \\
Hub ratio $\left(r_{h} / R\right)$ & 0.26 \\
Pitch ratio $(P / D)$ & 1 \\
Expanded area ratio $(E A R)$ & 0.6 \\
Skew angle [deg.] & Zero \\
Rake angle [deg.] & Zero \\
\hline
\end{tabular}

Table 2. Main dimensions of PODS.

\begin{tabular}{lc}
\hline Parameters & Value \\
\hline Maximum pod diameter ratio, Dpod/Dprop & 0.515 \\
Pod length ratio, Lpod/Dprop & 1.519 \\
Strut height ratio, Sheight/Dprop & 1.111 \\
Strut chord ratio, Schord/Dprop & 0.833 \\
Strut width ratio, Swidth/Dprop & 0.222 \\
Strut distance from propeller plan & $100 \mathrm{~mm}$ \\
Fore taper length & $85 \mathrm{~mm}$ \\
Fore taper angle & $15^{\circ}$ \\
Aft taper length & $110 \mathrm{~mm}$ \\
Aft taper angle & $25^{\circ}$ \\
\hline
\end{tabular}
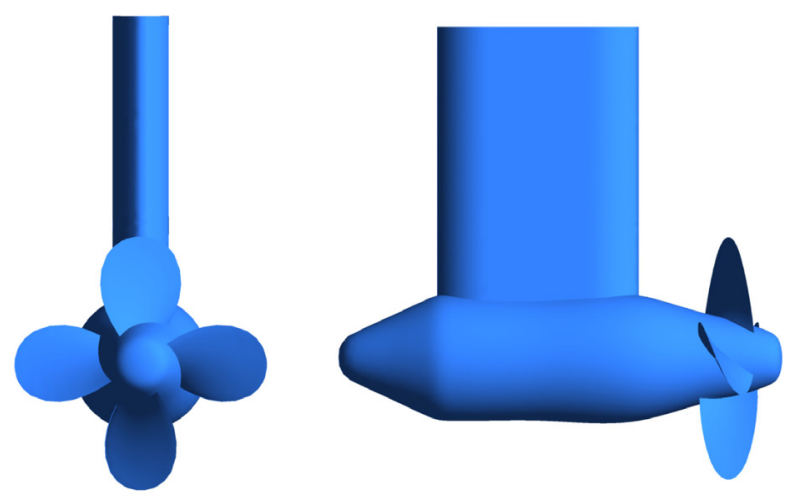

Fig. 9. Model of the PODS.

$(x, y, z)$ denote the upstream, starboard, and downward directions, respectively. The origin of the coordinate system is located at the center of the propeller hub.

\subsection{Single propeller}

This section presents a validation study of RANS and BEM predictions of a single propeller. Validation is made through the comparison of both numerical results against experimental test data of Liu [15] and Islam et al. [4]. These data are the result of a comprehensive experimental analysis of podded drive systems. In this study, particular test equipment was designed and several different measurements were also considered.

The numerical simulations for single propeller model are carried out in the same operating situation as the experimental conditions. Assuming the constant rotational

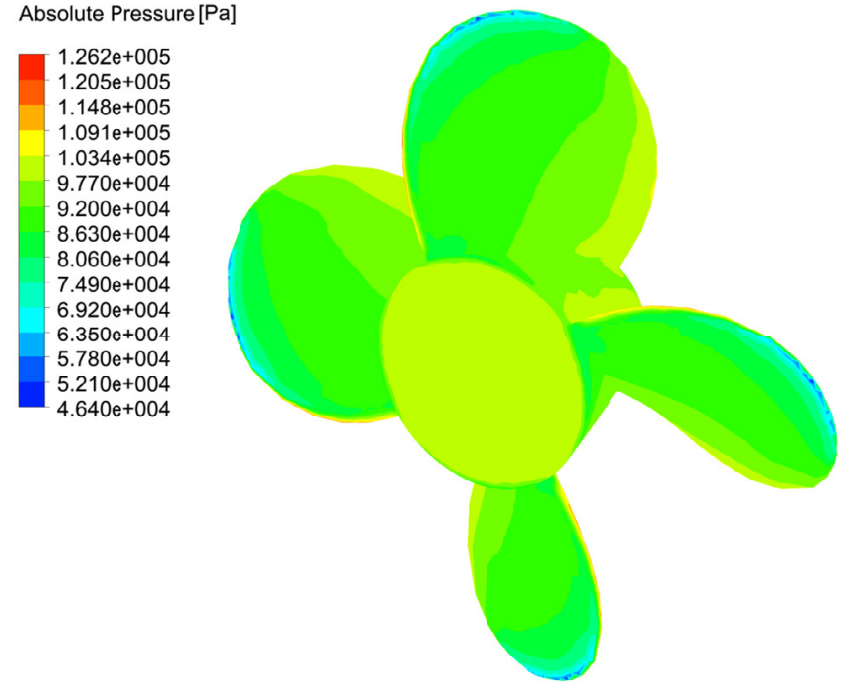

Fig. 10. Pressure distribution on the back side of the propeller at $J=0.2$.

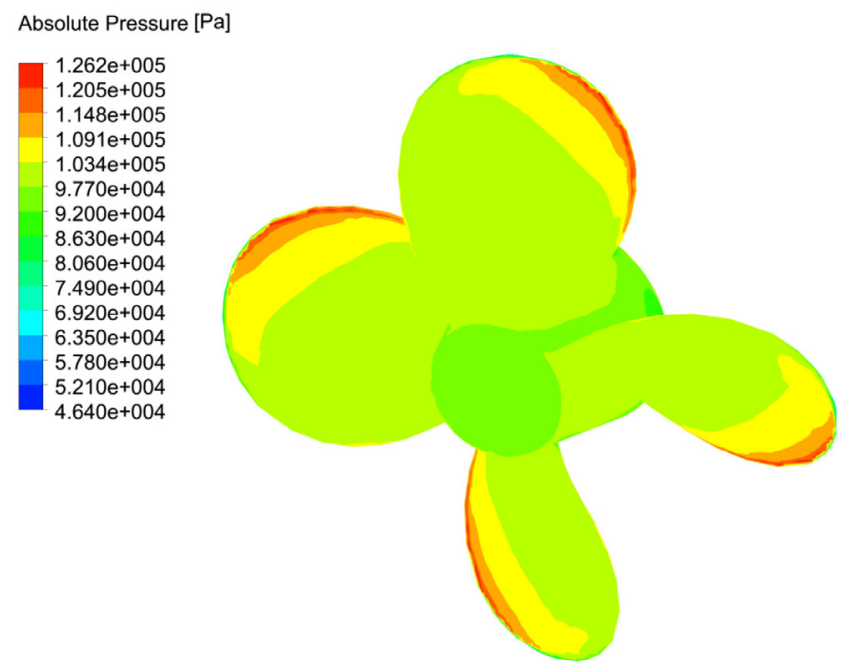

Fig. 11. Pressure distribution on the face side of the propeller at $J=0.2$.

velocity for the propellers, their performance is investigated for different inlet velocities to obtain the propulsive characteristics of the propellers in open water conditions. The rotational speed of the propellers is $15 \mathrm{rad} . \mathrm{s}^{-1}$. The thrust force and torque of the propellers are also computed for a range of advance velocity ratios from 0.2 to 1.0. Figures 10 and 11 represent the pressure distribution on the back and face sides of the propeller predicted with the RANS method at $J=0.2$, respectively. As observed in these figures, high pressure exists on the face and low pressure is present on the back side. The minimum pressures occur on the leading edge of the propeller blades and close to its tip. Quantitatively, the pressure distribution coefficient $\left(C_{P}=P / 0.5 \rho V_{R}^{2}\right.$, where: $\left.V_{R}^{2}=V_{A}^{2}+(2 \pi r n)^{2}\right)$ for the propeller at the blade root section and four different radii $(r / R=0.35,0.5,0.7$, and 0.9$)$ is shown 


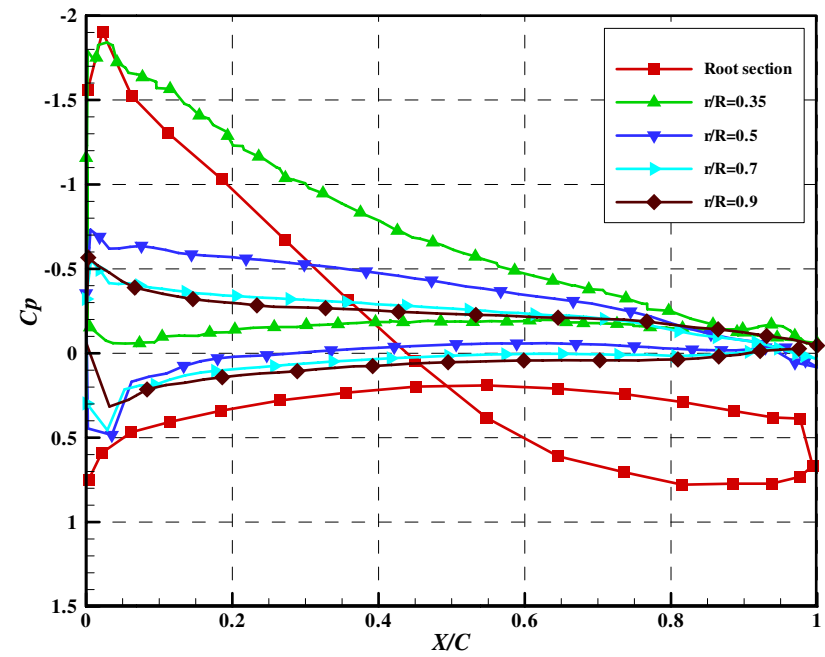

Fig. 12. Pressure coefficient distribution for st $J=0.2$.

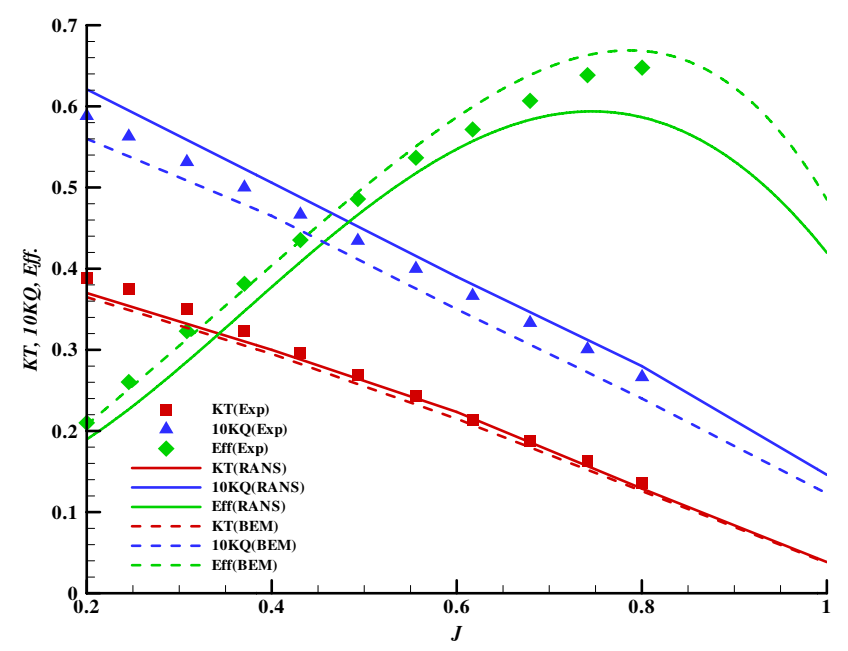

Fig. 13. Experimental and computational characteristic curves of the single propeller.

in Figure 12. The propeller has produced a good pressure distribution around the blade sections for thrust generation.

The predicted hydrodynamic characteristic curves obtained by BEM and RANS methods and their comparison with the experimental data for propeller model are presented in Figure 13. Within the achieved results, a fairly good agreement between the numerical results and the experimental data is observed. Furthermore, it is clear that in RANS simulations the propeller thrust coefficient is underestimated and propeller torque coefficient is overestimated, while in BEM calculations both propeller thrust and torque coefficient are underestimated.

\subsection{PODS}

Hydrodynamic performance for the PODS is also evaluated using BEM and RANS solver. The simulations are
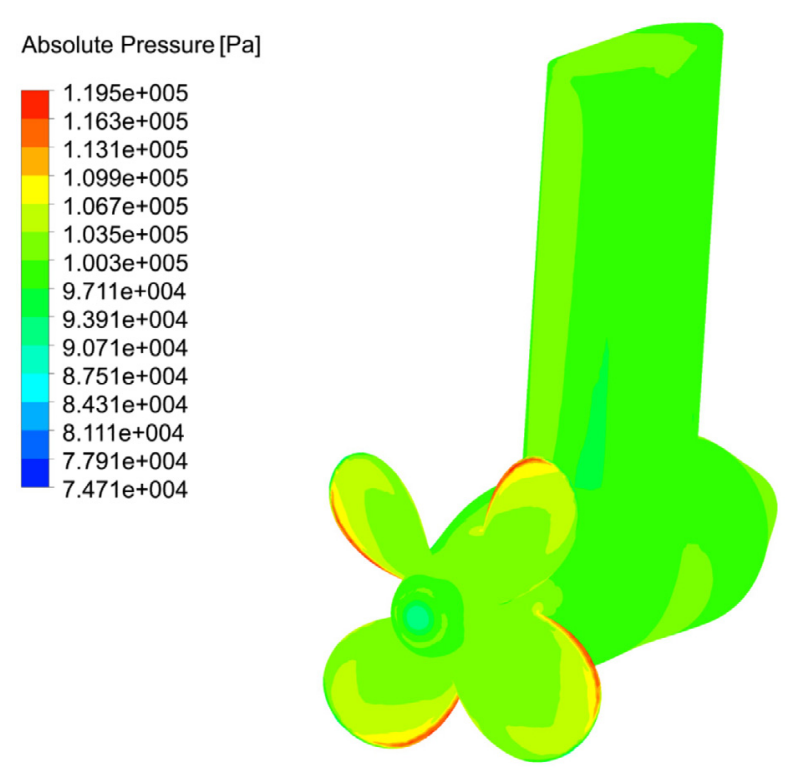

Fig. 14. Pressure distribution on the PODS and face side of the propeller at $J=0.6$.

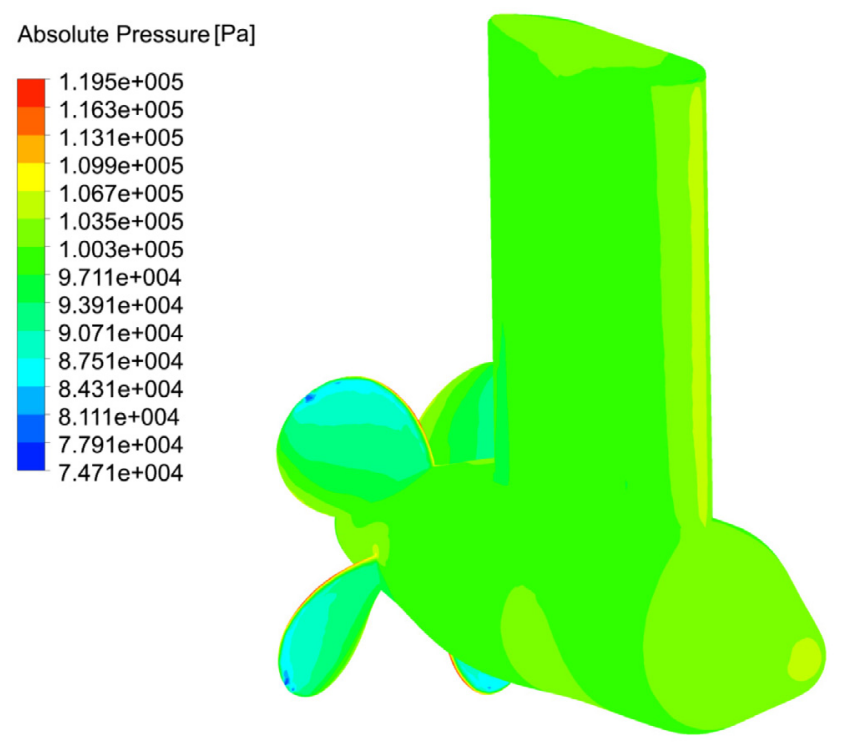

Fig. 15. Pressure distribution on the PODS and back side of the propeller at $J=0.6$.

performed for a range of advance velocity ratios from 0.2 to 1.0. These investigations are performed for pusher podded propulsor configuration. The total forces on the unit in each direction and propeller thrust and torque are computed. Figures 14 and 15 show pressure distribution on the pod, the strut, and the propeller predicted with the RANS method at an advance velocity ratio of $J=0.6$. In the pusher PODS, the propeller works in the wake of the strut. The cross flows from the pod and strut have both tangential and radial velocities leading to a strongly non-uniform inflow for the propeller. 


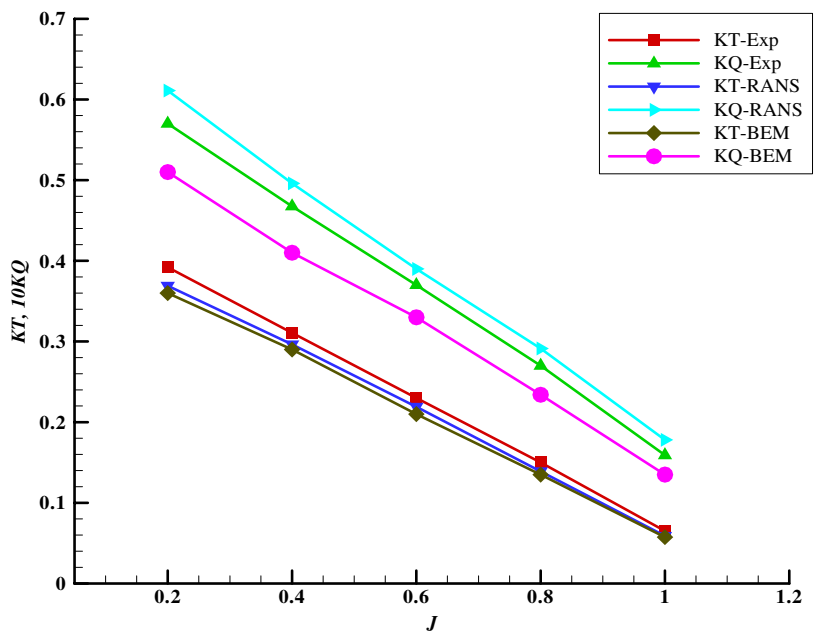

Fig. 16. Propeller thrust and torque coefficient.

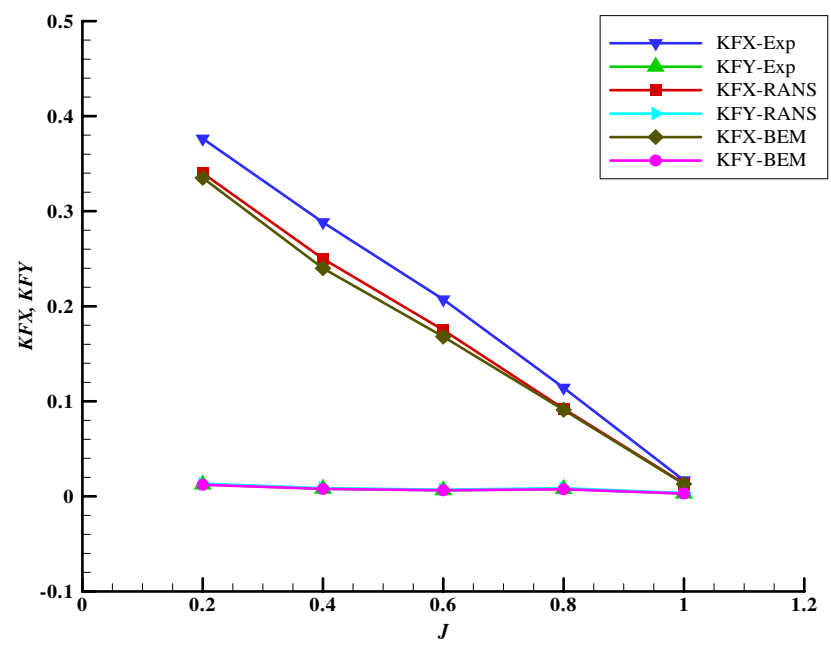

Fig. 17. Axial force and side force coefficient.

The PODS coefficients include the propeller thrust and torque coefficients and the axial coefficients as well as the side force coefficients for the pusher PODS in straight conditions are presented and compared to the experimental results in Figures 16 and 17. The propeller thrust and torque predictions for all advance velocity ratios are in overall agreement with the experimental data. The propeller thrust and torque variations at the higher advance velocity ratios is more evident than those seen at lower velocity ratios. Also, the discrepancy between BEM results and the experimental data is greater than for RANS results. The average error of $K_{T}$ and $K_{Q}$ errors for RANS results are less than $-6.5 \%$ and $+7.5 \%$, respectively. In BEM calculations, the average errors of $K_{T}$ and $K_{Q}$ are increased to $-9 \%$ and $-2.5 \%$.

In this study, the RANS simulation is carried out on a sixteen processor cluster of AMD Opteron Processors $6134(2.3 \mathrm{GHz})$ and total 32GB RAM. The BEM calculation is also performed on the single processor PC. The computational times for all advance velocity ratios are about $30 \mathrm{~min}$ and $10 \mathrm{~h}$ for the BEM and RANS solvers, respectively. Although BEM solver is a powerful tool for the design stage of the marine propeller and PODS, RANS solver has a greater capability in the prediction of propulsive characteristics for the optimization stage of the PODS. Unsteady RANS could be used in the future research. The error of propulsive characteristics could be reduced by applying this method, despite the fact that unsteady calculations require a great deal of computing time.

\section{Conclusions}

In this paper, a finite volume based RANS solver and a BEM code have been used to evaluate the performance of the single propeller and PODS. Validation is made through the comparison of both numerical results against experimental test data. Propulsive characteristics of the single propeller prove that the RANS and BEM predictions for all advance velocity ratios are in quite good agreement with the experimental data. These methods can well predict the propeller thrust and torque coefficient at different advance velocity ratios. The discrepancy between numerical and experimental results for the BEM code is more than that of the RANS solver. However, the differences between the potential and viscous results for the propeller thrust and torque coefficients are small and negligible and BEM method shows an acceptable accuracy. The study proves that the BEM method is an appropriate numerical scheme and a powerful tool for hydrodynamic analysis of the conventional marine propellers.

The numerical investigation of the PODS indicates that the propeller thrust and torque predictions for all advance velocity ratios are in overall agreement with the experimental data. It is found that the results predicted by the RANS method are closer to the experimental data than for the BEM method. Based on the result of simulations, the relative errors for axial force and side force coefficient in BEM calculation are greater than for RANS solver. This discrepancy between the numerical results could be probably related to the interaction between the propeller, pod, and strut. In PODS the viscous force contributes a great part in the axial and the side force. Inviscid flow solvers have a limitation in terms of the correct prediction of the hydrodynamic performance of the PODS. In BEM simulations the effect of the pod and the strut viscous drag are not considered. BEM with corrections for the drag of the pod and the strut should lead to closer comparison with the experiments. Due to the lower computational time and efficient grid generation, BEM method is applicable to the early stage of the design of the marine propeller and PODS. Moreover, RANS solver is necessary for optimization stage of the PODS.

Acknowledgements. The work presented in this paper has been supported by the High Performance Computing Research Center (HPCRC) at Amirkabir University of Technology (AUT). The authors gratefully like to thank the reviewers for their comments that helped us to improve the manuscript. 


\section{References}

[1] J.S. Carlton, Marine propeller and propulsion, Butterworth-Heinemann Ltd, 2013

[2] R. Shamsi, H. Ghassemi, Hydrodynamic analysis of puller and pusher of azimuthing podded drive at various yaw angles, Proceedings of the Institution of Mechanical Engineers, Part M: J. Eng. Maritime Environment 228 (2014) 55-69

[3] H. Ghassemi, P. Ghadimi, Hydrodynamic Efficiency Improvement of the High Skew Propeller for the Underwater Vehicle Under Surface and Submerged Conditions, J. Ocean Univ. China 10 (2011) 314-324

[4] M.F. Islam, B. Veitch, A. Akinturk, N. Bose, P. Liu, Performance study of podded propulsor in static azimuthing conditions, Int. Shipbuilding. Progress. 56 (2009) 135-157

[5] P. Liu, M. Islam, B. Veitch, Unsteady hydromechanics of a steering podded propeller unit, Ocean Eng. 36 (2009) 1003-1014

[6] C.Y. Guo, N. Ma, C.J. Yang, Numerical simulation of a podded propulsor in viscous flow, J. Hydrodynamics 21 (2009) 71-76

[7] H. Amini, L. Sileo, S. Steen, Numerical calculations of propeller shaft loads on azimuth propulsors in oblique inflow, J. Marine Sci. Technol. 17 (2012) 403-421

[8] Y. Arikan, A. Doğrul, F. Çelik, Performance analysis and investigation of the slipstream flow of podded propeller, Brodogradnja 63 (2012) 226-233
[9] R. Shamsi, H. Ghassemi, D. Molyneux, P. Liu, Numerical Hydrodynamic Evaluation of Propeller (with Hub Taper) and Podded Drive in Azimuthing Conditions, J. Ocean Eng. 76 (2013) 121-135

[10] R. Shamsi, H.Ghassemi, Time-Accurate Analysis of the Viscous Flow Around Puller Podded Drive Using Sliding Mesh Method, ASME J. Fluids Eng. 137 (2015) 1-9

[11] X. Chang, J. Zou., S. Huang, C. Guo, Influence on the hydrodynamic performance of a variable vector propeller of different rules of pitch angle change, J. Marine Sci. Appl. 6 (2007) 32-36

[12] H. Ghassemi, M. Ikehata, H. Yamasaki, An Investigation of Wake Model and Its Effect on Hydrodynamic Performance of Propellers by Using a Surface Panel Method, J. Society Naval Architects Japan 178 (1995) 83-91

[13] D.C. Wilcox, Turbulence modeling for CFD, 3rd edition, DCW Industries, Inc. La Canada, California, 2006

[14] H. Ghassemi, P. Ghadimi, Computational hydrodynamic analysis of the propeller rudder and the AZIPOD systems, Ocean Eng. 35 (2008) 117-130

[15] P. Liu, The Design of a Podded Propeller Base Model Geometry and Prediction of its Hydrodynamics, Institute for Ocean Tech. National Research Council, Canada. (TR-2006-16), 2006 